\title{
BMJ Open Quality Catheter-associated urinary tract infection reduction in critical care units: a bundled care model
}

\author{
Stephanie Grana Van Decker, ${ }^{1}$ Nicholas Bosch, ${ }^{2}$ Jaime Murphy ${ }^{2}$
}

To cite: Van Decker SG, Bosch N, Murphy J. Catheterassociated urinary tract infection reduction in critical care units: a bundled care model. BMJ Open Quality 2021;10:e001534. doi:10.1136/ bmjoq-2021-001534

Received 20 April 2021 Accepted 26 November 2021

Check for updates

(c) Author(s) (or their employer(s)) 2021. Re-use permitted under CC BY-NC. No commercial re-use. See rights and permissions. Published by BMJ.

${ }^{1}$ Department of Internal Medicine, Boston Medical Center, Boston, Massachusetts, USA

${ }^{2}$ Department of Pulmonary, Allergy, Sleep, and Critical Care Medicine, Boston Medical Center, Boston, Massachusetts, USA

Correspondence to

Dr Stephanie Grana Van Decker; svandec@gmail.com

\section{ABSTRACT}

Catheter-associated urinary tract infections (CAUTIs) represent approximately $9 \%$ of all hospital acquired infections, and approximately $65 \%-70 \%$ of CAUTIs are believed to be preventable. In the spring of 2013, Boston Medical Center (BMC) began an initiative to decrease CAUTI rates within its intensive care units (ICUs). A CAUTI taskforce convened and reviewed process maps and gap analyses. Based on Centers for Disease Control and Prevention (CDC) and Institute for Healthcare Improvement (IHI) guidelines, and delineated by the Healthcare Infection Control Practices Advisory Committee 2009 guidelines, all BMC ICUs sequentially implemented plan-do-studyact cycles based on which measures were most easily adaptable and believed to have the highest impact on CAUTI rates. Implementation of five care bundles spanned 5 years and included (1) processes for insertion and maintenance of foley catheters; (2) indications for indwelling foley catheters; (3) appropriate testing for CAUTIs; (4) alternatives to indwelling devices; and (5) sterilisation techniques. Daily rounds by unit nursing supervisors and inclusion of foley catheter necessity on daily ICU checklists held staff accountable on a daily basis. With these interventions, the total number of CAUTIs at BMC decreased from 53 in 2013 to 9 in 2017 (83\% reduction) with a $33.8 \%$ reduction in indwelling foley catheter utilisation during the same time period. Adapted protocols showed success in decreasing the CAUTI rate and indwelling foley catheter usage in all of the BMC ICU's. While all interventions had favourable and additive trends towards decreasing the CAUTI rate, the CAUTI awareness education, insertion and removal protocols and implementation of PureWick female incontinence devices had clear and significant effects on decreasing CAUTI rates. Our project provides a framework for improving HAls using rapid cycle testing and U-chart data monitoring. Targeted education efforts and standardised checklists and protocols adapted sequentially are low-cost and high yield efforts that may decrease CAUTIs in ICU settings.

\section{PROBLEM}

Healthcare-associated infections (HAIs) are a significant cause of morbidity and mortality in hospitalised patients in the USA. ${ }^{1}$ Catheterassociated urinary tract infections (CAUTIs), urinary tract infections that occur in the context of an indwelling foley catheter, represent approximately $9 \%$ of all HAIs,${ }^{2}$ and are associated with increased morbidity, mortality and cost in intensive care units (ICUs). ${ }^{3-5}$ Given that $65 \%-70 \%$ of CAUTIs may be preventable ${ }^{6}$ they are the target of ongoing national $^{7}$ and international ${ }^{58}$ preventable harm initiatives to improve patient safety and cut costs in the healthcare delivery system. In an effort to reduce the rate of ICU CAUTIs at our hospital, we serially implemented bundled CAUTI-prevention tools and measured the effect of the implementation on CAUTI rates in the ICU. In this manuscript, we describe our experiences using bundled CAUTI-prevention tools in a tertiary care, inner city, safety net, medical centre.

\section{BACKGROUND}

Boston Medical Center (BMC) is a 487-bed safety-net hospital in Boston, Massachusetts. ${ }^{9} 10$ In accordance with the Centers for Medicare and Medicaid Services Hospital Inpatient Quality Reporting mandate, BMC reports CAUTI rates to the National Healthcare Safety Network (NHSN). The NHSN provides hospitals with real-time data on their HAI performance through various measures including the Standardized Infection Ratio (SIR) ${ }^{11}$ an adjusted index allowing HAI rate comparison between US hospitals. ${ }^{12}$ In the spring of 2013, the SIR for CAUTIs among BMC ICUs was $65 \%$ greater than predicted. Consequently, BMC formed a multidisciplinary CAUTI task force to implement dataproven CAUTI-reduction techniques based on current best practices and guidelines, and monitored effects of the interventions longitudinally. The formation of the Executive Critical Care Committee in 2011 by hospital leadership established the groundwork for collaboration between all the BMC ICUs and among the key disciplines involved in ICU decision-making, including pharmacy, nursing and physicians. The CAUTI taskforce aimed to reduce CAUTIs by $10 \%-20 \%$ and to reduce the BMC SIR for CAUTIs to the national average. 


\section{BASELINE MEASUREMENT}

CAUTIs were defined according to the published CDC definition ((1) use of an indwelling foley for more than two consecutive days, (2) catheter in place on day of or day prior to event, (3) two or more symptoms concerning for CAUTI and (4) urine culture with no more than two species of organism of which at least one is a bacterium of $\geq 105 \mathrm{CFU} / \mathrm{mL}$ ). ${ }^{13}$ CAUTIs were captured in real time using medical chart surveillance. CAUTI rate, rather than SIR, is readily trackable at $\mathrm{BMC}$ in real time, and thus was chosen as the best measure to monitor the CAUTI reduction tools used. In addition, during the intervention, NHSN changed the definitions of CAUTIs resulting in challenges using SIR and NHSN percentiles to compare preintervention and postintervention data. Prior to the planned interventions, the baseline ICU CAUTI rate was $\mathbf{5 . 8 6}$ per 1000 ICU patient-days with a corresponding SIR of 1.65 .

\section{DESIGN}

The CAUTI taskforce is composed of key stakeholders including representatives from the infection prevention department, quality and patient safety department, ICU nurse managers, ICU nurse educators, urologists and intensivists. We plotted the current workflow for foley catheter management and CAUTI identification using process maps, which we then used to perform gap analyses. Gap analyses identified that
BMC processes diverged from national guidelines, especially with regard to infection prevention tactics and CAUTI identification. Subsequently, the CAUTI taskforce created driver diagrams based on clinician expertise to delineate key strategies for improvement in CAUTI rates and conducted preventability analyses on identified CAUTIs (figures 1 and 2).

Based on CDC and IHI guidelines, and delineated by the Healthcare Infection Control Practices Advisory Committee 2009 guidelines,${ }^{14}$ we implemented new practices sequentially in plan-do-study-act (PDSA) cycles starting with measures that we deemed most easily adaptable and most likely positively impact CAUTI rates. The CAUTI taskforce drafted interventions based on available guidelines and then distributed intervention protocols to stakeholders via email listserv, ICU paper postings, departmental meetings and institutional intranet postings. In preparation for implementation, nurses completed simulation and web-based training programmes. In collaboration with the institutional Information Technology Department, the CAUTI taskforce developed electronic best practice advisories (BPAs) within the Epic electronic medical record.

Given the planned sequential implementation and varied stakeholders involved, the CAUTI taskforce created a Microsoft Outlook distribution group, ('DG-CAUTI'), where clinicians and support staff could email suggestions for improvement of piloted interventions, ask questions regarding the ongoing CAUTI-reduction efforts

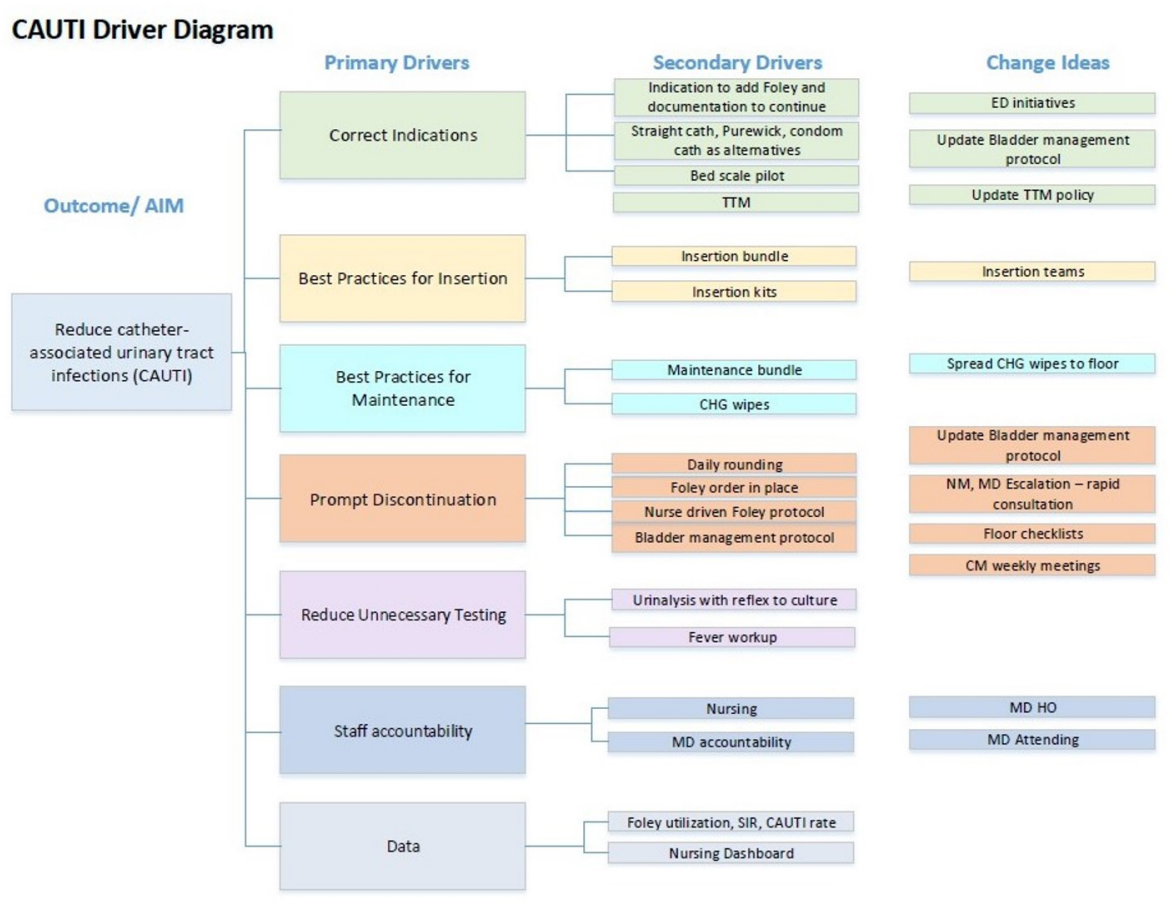

Figure 1 CAUTI driver diagram. Driver diagram depicting primary aim of CAUTI task force as well as proposed primary and secondary drivers targeted to achieve this aim. Depicted are interventions implimented as part of the CAUTI bundle as well as those that were reviewed and fall outside of the purview of this intervention. $\mathrm{CHG}$, chlorhexidine gluconate. TTM, targeted temperature management; NM, nurse manager; MD, physician; HO, house officer; CM, case management; SIR, standard infection ratio. 


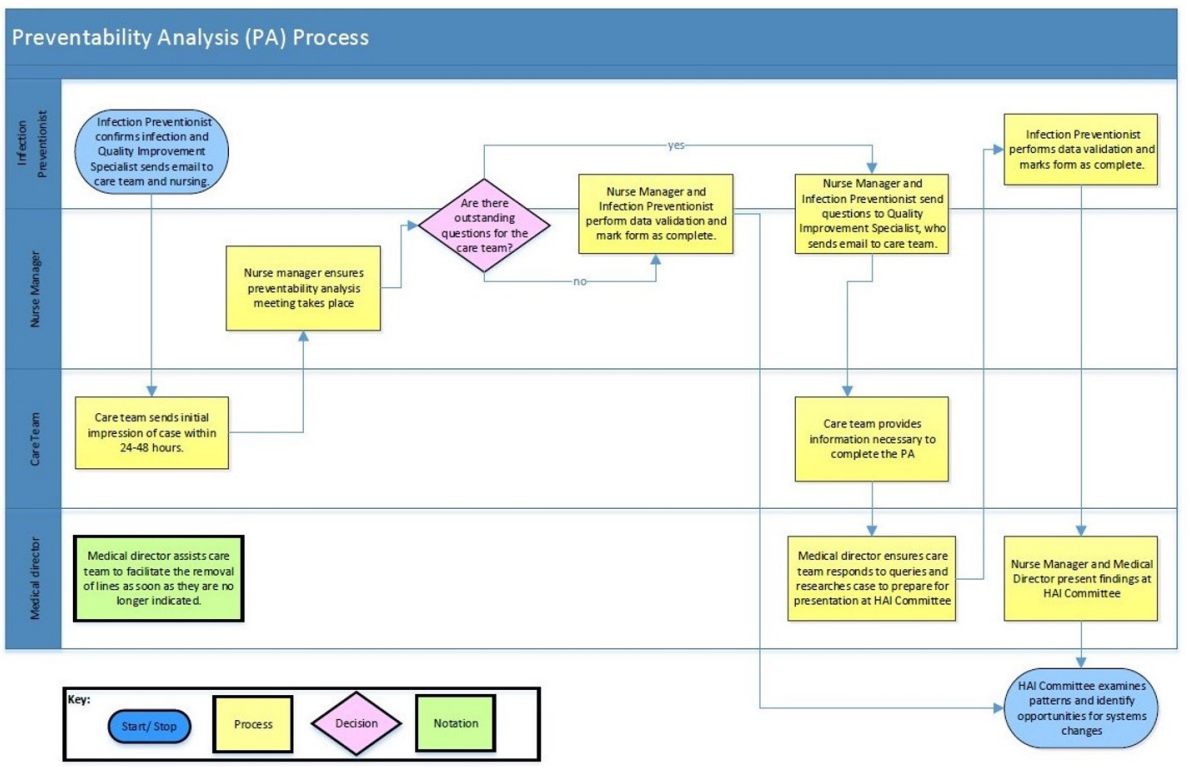

Figure 2 Catheter-associated urinary tract infection (CAUTI) preventability analysis (PA). Process by which the CAUTI taskforce identified CAUTIs is depicted. Root cause analyses subsequently undertaken to develop designed interventions. HAl, healthcare-associated infection.

and where CAUTI taskforce members could clarify device use and protocols.

\section{STRATEGY}

The CAUTI taskforce used the PDSA cycle framework to serially implement initiatives aimed at reducing CAUTI rates. We plotted CAUTI rates monthly and evaluated the impact of each PDSA cycle using a standard run chart. Once we had a sufficient number of data points (ie, 20) we switched to a statistical process control chart (SPC). SPC charts are preferable to run charts as they have upper and lower control limits which more readily allow for discrimination of special causes from common cause variation. A U-chart, a specific type of SPC chart, was used because it accurately analyses data in which it is possible for an individual patient to have more than one defect per unit (ie, more than one CAUTI per episode of foley catheter) and to account for variable subgroup size (ie, variance in patient volume each month).

\section{PDSA cycle 1: didactic and simulation-based education} initiative to increase awareness of CAUTIs and standardised foley catheter insertion and maintenance protocols (September 2014; project month 0)

The CAUTI task force created nursing and physician education courses targeting preventability of CAUTIs and appropriate insertion and maintenance techniques of indwelling foley catheters. Designated department quality leaders in surgical specialties and ICU nurse managers scheduled formal education on foley catheter insertion and maintenance for staff. Materials used during these trainings closely paralleled the Healthcare Infection Control Practices Advisory Committee 2009 guidelines $^{14}$ and were distributed in May 2014 with the goal of official implementation by September 2014. Physicians and nurses completed foley catheter insertion and maintenance in-person simulation training. Additionally, nursing staff completed mandatory relevant online modules through the hospital nursing education portal, received retraining on foley catheter insertion processes at an annual competency day, and received 1:1 or small group bedside education according to standard ICU practices.

\section{PDSA cycle 2: evidence-based foley catheter use (January} 2016, project month 16)

The CAUTI taskforce created and published a list of hospital-approved indications for indwelling urinary devices, which they based on national guidelines ${ }^{14}$ and had reviewed by the urology department for their specialtyspecific indications for bladder management. We posted the indications and appropriate foley catheter insertion and maintenance techniques, titled 'Bladder Management Protocol', on the local intranet for ease of access. Nursing managers and department leaders encouraged providers and staff to remove foley catheters when no longer meeting indications. To support this effort, nurse managers used an EMR-generated daily foley catheter report to identify patients with indwelling catheters and review if they met criteria to retain them. Feedback via the DG-CAUTI listserv as well as to nurse managers and CAUTI taskforce members facilitated continuous refinement of acceptable indications for foley catheter use in critically ill patients.

PDSA cycle 3: urinalysis (UA) with reflex to culture (January 2016, project month 16)

Too often, patients are identified as having a CAUTI if they have an indwelling foley catheter, fever and positive 
urine culture with or without reportable symptoms. ${ }^{13}$ The literature demonstrates that a negative UA has an excellent negative predictive value for CAUTI in ICUs as well as prevent unwarranted antibiotic exposure in critically ill patients. ${ }^{15-17}$ Thus, the CAUTI taskforce created a BPA to encourage practitioners who ordered concurrent UA and urine culture to change their order to UA only and those who attempted to order urine cultures without a recent UA ( $<24$ hours) to cancel the culture order and instead opt for UA alone. The goal being to reduce the number of urine cultures sent on non-infectious UAs. Opt-out criteria for cancelling the simultaneous urine culture include neutropenia and septic shock. In order to reduce the likelihood of false positive culture results due to the presence of biofilms on indwelling foley catheters, ${ }^{17}$ patients with a positive UA had a new foley catheter placed prior to urine culture collection.

\section{PDSA cycle 4: PureWick female incontinence device (January 2017, project month 28)}

Professional organisations advocate for increased use of external urinary collection devices to prevent CAUTIs. ${ }^{18}$ Among male inpatients, external collection devices are associated with a lower incidence of symptomatic UTIs and bacteriuria than with indwelling devices. ${ }^{19}$ More recently, companies have developed external urinary collection devices for women. ${ }^{20}$ Recent reports suggest PureWicks provide a non-invasive method to manage female urinary incontinence, measure urinary output and reduce skin breakdown from urinary incontinence. ${ }^{21} 22$ Despite the early stages of this technology, BMC leadership felt it imperative to be on the forefront of the movement towards non-invasive urinary incontinence management, given the promising experiences of other institutions. The CAUTI taskforce implemented a protocol for the use of the PureWick external catheter among female ICU patients requiring strict output documentation, had an indwelling foley catheter for longer than 3 days, or those on bedrest and at risk for pressure injuries. The protocol implementation used simulation and online modules consistent with the methods outlined in cycle 1 .

\section{PDSA cycle 5: chlorhexidine gluconate (CHG) bathing (June} 2017, project month 33)

CHG baths reduce drug-resistant infections and blood stream infections from the various life-support equipment used in ICUs. ${ }^{23-25}$ Independent, but complimentary to the efforts of the CAUTI taskforce, separate hospital groups tasked to evaluate and reduce the number of catheter-associated central line infections trialled CHG baths for all ICU patients. Education for nursing staff on how to perform CHG baths used simulation and online module tools consistent with the methods outlined in Cycle 1. While the initial reduction in CAUTI rates with CHG baths showed promise, we halted the practice for 2 weeks in December 2017 following a notable elevation in CAUTI rate. Investigation by the CAUTI taskforce found the special cause variation correlated with the several logistical difficulties, including compatibility of wipes with hospital infrastructure, coinciding with the holiday season, when BMC typically sees a slight increase in CAUTI. Once identified and remediated, the CAUTI taskforce re-initiated CHG baths and noted a continued downtrend in CAUTI rates as compared with prior to this practice's implementation.

\section{RESULTS}

With the above listed interventions, the total number of CAUTIs at BMC decreased from 53 in 2013 to 9 in 2017 (83\% reduction) and a $33.8 \%$ reduction in indwelling foley catheter utilisation from the fourth quarter of 2015 to the fourth quarter of 2017. BMC had a mean CAUTI rate of 5.86 per $1000 \mathrm{ICU}$ patient-days prior to the above listed interventions.

We mapped CAUTI rates and intervention time using a U-chart (figure 3). The data plotted showed a significant decrease in CAUTI rate associated with the CAUTI awareness campaign and with the new foley catheter insertion and maintenance protocols implemented in September 2014. These educational efforts decreased the mean CAUTI rate to 3.25 per 1000 ICU patient-days, suggesting the creation of a new sustainable system. Subsequently, the concurrent implementation of the 'Bladder Management Protocol' and UA with reflex to culture resulted in decreased CAUTI rates, however, did not manage to create a new system as evidenced by the U-chart. The CAUTI rate in the quarter preceding implementation of these processes was 2.48 (fourth quarter 2015) and following these interventions was 2.24 in the first quarter of 2016. The implementation of PureWick catheter resulted in a significant downward shift in the mean CAUTI rate to 1.62 suggesting the creation of a new system with intrinsically lower CAUTI rate. The CHG bathing protocol initiation correlated with a downtrend in CAUTI rates with a preimplementation rate of 1.27 in the second quarter of 2017 and postimplementation rate of 0.61 in the fourth quarter of 2017. The hospital encountered several challenges in the implementation of this intervention and noted special cause variation in the U-chart that required cessation of practice for 2 weeks in January 2018 for investigation. Eventually we reinstituted the practice with continued improvement of CAUTI rate reduction.

\section{DISCUSSION}

Our multi-disciplinary CAUTI taskforce reviewed institutional policies, documented deficiencies, and initiated rapid cycle testing in the form of PDSA cycles to improve CAUTI rates. PDSA cycles built off each other, with new interventions implemented as soon as the CAUTI taskforce finalised the protocols. Protocols showed success in decreasing the CAUTI rate and indwelling foley catheter usage in all of the BMC ICU's. While all interventions had favourable and additive trends towards decreasing the CAUTI rate, the CAUTI awareness education, insertion 


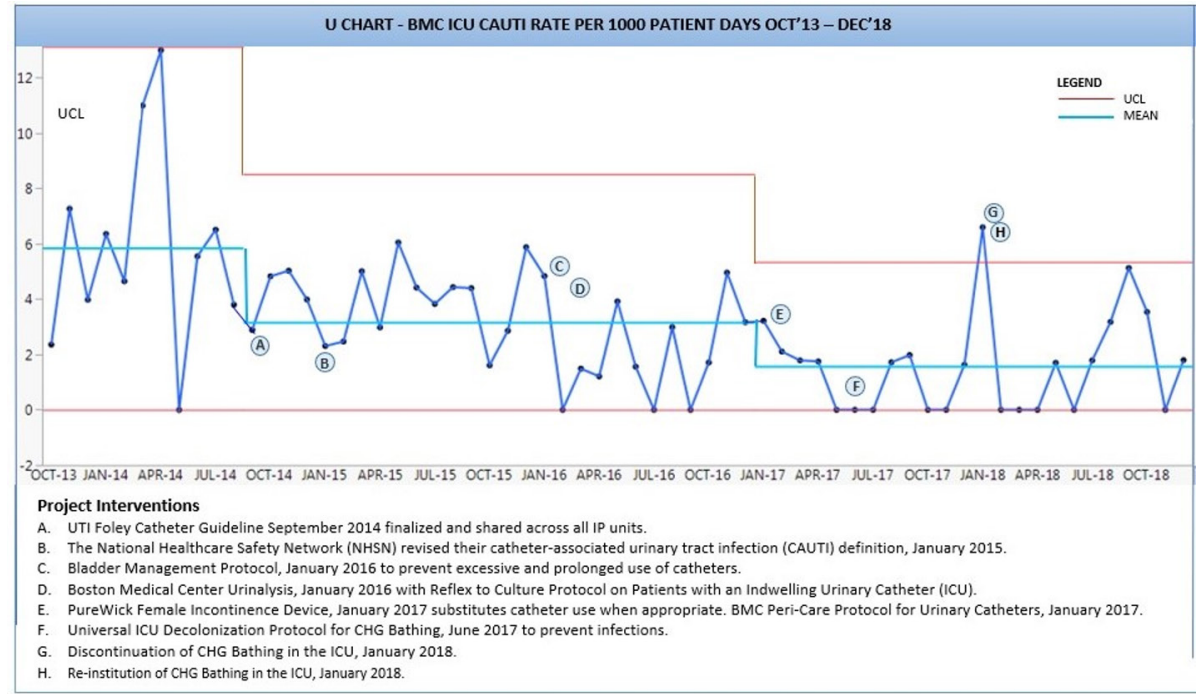

Figure 3 Boston Medical Center (BMC) CAUTI rate per 1000 patient-days October 2013 to December 2018. U-chart depicting CAUTI rate decline in response to rapid cycle testing of CAUTI bundle with a significant decrease in rate of CAUTIs with the CAUTI awareness campaign and with the new indwelling foley catheter insertion and maintenance protocols implemented in September 2014 as well as implementation of PureWick catheter. CHG, chlorhexidine gluconate; ICU, intensive care unit; UCL, upper control limit; UTI, urinary tract infection; IP; inpatient.

and removal protocols, and implementation of PureWick female incontinence devices had clear and significant effects on decreasing CAUTI rates. Our project provides a framework for improving HAIs using rapid cycle testing and U-chart data monitoring. We identified and confirmed several CAUTI-reduction methods that successfully reduce CAUTI rates in ICUs.

Our findings mirror those of several other institutions who aimed to reduce CAUTI rates in both ICUs and on medical and surgical floors with bundled care initiatives. ${ }^{26-35}$ We concur with the previous studies that clear foley catheter indication guidelines, insertion and pericare processes, and appropriate testing can effectively yield lower CAUTI rates. Institutional prioritisation of CAUTI reduction and continued education of frontline staff on approved protocols remain excellent ways to reduce infection rates. This is the first published study using U-charts to track and display CAUTI reduction effects, as compared with run charts and alternative control charts. We believe U-charts most informatively portray the trend of CAUTI rate as well as allow for interpretation of special cause effect. We demonstrated significant improvement in CAUTI rates with the implementation of PureWick catheters, which to date have been sparsely studied. ${ }^{36-38}$ Further studies investigating the effects of external urinary collection devices for female patients, including PureWicks, is warranted. Despite reaching our intended outcomes, which included reduction in foley use and decreased CAUTI rates, we did not specifically design our intervention to quantitatively assess staff knowledge or the impact of our educational efforts on staff behaviour. Thus, we cannot exclude that the improvement in CAUTI rates were due to other factors. Unexpected hurdles included the implementation of the CHG bath protocol.
Trial of any new devices or wipes should go through a hospital's standardised instrument trialling process.

BMC's success stemmed in part to the leadership of the Executive Critical Care Committee and the standardisation of processes among all BMC's ICUs. Thus, the generalisability of BMC's results are in a sense limited by this committee, as institutions without similar crossdepartmental buy-in may be less likely to reproduce these results. The culture at BMC, stimulated by the Executive Critical Care Committee, focuses on strong interprofessional collaboration. This culture stimulates an open dialogue and culture of safety across all the ICUs.

\section{CONCLUSION}

Over the course of 4 years, rapid cycle testing of evidencebased interventions focused on education and protocolisation of indwelling foley catheter management and CAUTI diagnosis with PDSA cycles improved CAUTI rates at BMC. These interventions achieved a sustained reduction in CAUTIs at BMC and reduced the foley catheter utilisation rate. Strong inter-ICU and interprofessional collaboration to standardise and optimise processes among the ICUs was critical to the success of our CAUTI quality improvement programme. Further studies are needed to compare external urinary collection devices for females and each practice setting will need to investigate site-specific requirements with regard to CHG bathing given our challenges as described above. However, despite the obstacles, BMC faced in targeted education efforts and standardised checklists and protocols adapted sequentially are low-cost and high-yield efforts that may decrease CAUTIs in ICU settings. 
Acknowledgements Thank you to Deeksha Ahluwalia, MPH for providing statistical support and figure design of key figures included in the manuscript.

\section{Collaborators Deeksha Ahluwalia, MPH}

Contributors JM played an integral role in the study design, assisted stakeholders with evaluation of evidence-based practices and implementation of chosen practices, contributed to editing of manuscript and provided statistical and study design expertise. JM is the guarantor of this publication. NB provided statistical expertise and study design expertise and was critical to the editing of manuscript. SGVD performed data analysis and wrote and edited manuscript. All authors contributed to and approved the final manuscript.

Funding The authors have not declared a specific grant for this research from any funding agency in the public, commercial or not-for-profit sectors.

Competing interests None declared.

Patient and public involvement Patients and/or the public were not involved in the design, or conduct, or reporting, or dissemination plans of this research.

Patient consent for publication Not applicable.

Ethics approval This study does not involve human participants.

Provenance and peer review Not commissioned; externally peer reviewed.

Data availability statement Data are available upon request.

Open access This is an open access article distributed in accordance with the Creative Commons Attribution Non Commercial (CC BY-NC 4.0) license, which permits others to distribute, remix, adapt, build upon this work non-commercially, and license their derivative works on different terms, provided the original work is properly cited, appropriate credit is given, any changes made indicated, and the use is non-commercial. See: http://creativecommons.org/licenses/by-nc/4.0/.

\section{REFERENCES}

1 Centers for Disease Control and Prevention. Guideline for prevention of catheter-associated urinary tract infections (2009), 2015. Available: https://www.cdc.gov/infectioncontrol/guidelines/cauti/background. html

2 Magill SS, Edwards JR, Bamberg W, et al. Multistate pointprevalence survey of health care-associated infections. N Engl J Med 2014:370:1198-208.

3 Nicolle LE. Catheter associated urinary tract infections. Antimicrob Resist Infect Control 2014;3:23.

4 Hollenbeak CS, Schilling AL. The attributable cost of catheterassociated urinary tract infections in the United States: a systematic review. Am J Infect Control 2018;46:751-7.

5 Rosenthal VD, Dwivedy A, Calderón MER, et al. Time-Dependent analysis of length of stay and mortality due to urinary tract infections in ten developing countries: INICC findings. J Infect 2011;62:136-41.

6 Umscheid CA, Mitchell MD, Doshi JA, et al. Estimating the proportion of healthcare-associated infections that are reasonably preventable and the related mortality and costs. Infect Control Hosp Epidemiol 2011;32:101-14

7 Centers for Medicare and Medicaid Services (CMS), HHS. Medicaid program; payment adjustment for provider-preventable conditions including health care-acquired conditions. final rule. Fed Regist 2011;76:32816-38.

8 Rosenthal VD. Device-Associated nosocomial infections in limitedresources countries: findings of the International nosocomial infection control Consortium (INICC). Am J Infect Control 2008;36:S171.e7-S171.e12.

9 Boston University Medical Center. GME diversity \& inclusion: committment to the community, 2019. Available: https://www.bmc. $\mathrm{org} /$ medical-professionals/office-minority-physician-recruitment

10 Boston Medical Center. Bmc facts, 2017. Available: https://www. bmc.org/sites/default/files/For_Medical_Professionals/BMC-Facts. pdf

11 Centers for Disease Control and Prevention. Operational guidance for acute care hospitals to report catheter-associated urinary tract infection (CAUTI) data to CDC's NHSN for the purpose of fulfilling CMS's hospital inpatient quality reporting (IQR) requirements, 2014. https://www.cdc.gov/nhsn/pdfs/final-ach-cauti-guidance.pdf

12 NHSN. The NHSN standardized infection ratio (Sir): a guide to the Sir, 2019. https://www.cdc.gov/nhsn/pdfs/ps-analysis-resources/nhsnsir-guide.pdf

13 Centers for Disease Control and Prevention. Urinary Tract Infection (Catheter-Associated Urinary Tract Infection [CAUTI] and Non-
Catheter-Associated Urinary Tract Infection [UTI]) and Other Urinary System Infection [USI]) Events, 2019.

14 Gould CV, Umscheid CA, Agarwal RK, et al. Guideline for prevention of catheter-associated urinary tract infections 2009. Infect Control Hosp Epidemiol 2010;31:319-26.

15 Stovall RT, Haenal JB, Jenkins TC, et al. A negative urinalysis rules out catheter-associated urinary tract infection in trauma patients in the intensive care unit. J Am Coll Surg 2013;217:162-6.

16 Lee SP, Vasilopoulos T, Gallagher TJ. Sensitivity and specificity of urinalysis samples in critically ill patients. Anaesthesiol Intensive Ther 2017;49:204-9.

17 Guide AA. Guide to the elimination of catheter-associated urinary tract infections (CAUTIs). developing and applying Facility-Based prevention interventions in acute and long-term care settings, 2008. https://oeps.wv.gov/cauti/Documents/CAUTI Guide_APIC.pdf

18 Gray M, Skinner C, Kaler W. External collection devices as an alternative to the indwelling urinary catheter: evidence-based review and expert clinical panel deliberations. J Wound Ostomy Continence Nurs 2016;43:301-7.

19 Saint S, Kaufman SR, Rogers MAM, et al. Condom versus indwelling urinary catheters: a randomized trial. J Am Geriatr Soc 2006;54:1055-61.

20 Beeson T, Davis C. Urinary management with an external female collection device. J Wound Ostomy Continence Nurs $2018 ;$;45:187-9. Mar/Apr.

21 Bleeker E, Koehlmoos A, Glover E. External catheters and reducing adverse effects in female inpatients, 2019. Available: https:// nwcommons.nwciowa.edu/cgi/viewcontent.cgi?article $=1026 \&$ context=celebrationofresearch

22 Eckert L, Mattia L, Patel S, et al. Reducing the risk of indwelling catheter-associated urinary tract infection in female patients by implementing an alternative female external urinary collection device: a quality improvement project. $J$ Wound Ostomy Continence Nurs 2020;47:50-3.

23 Frost SA, Alogso M-C, Metcalfe L, et al. Chlorhexidine bathing and health care-associated infections among adult intensive care patients: a systematic review and meta-analysis. Crit Care 2016;20:379.

24 Huang $\mathrm{H}-\mathrm{P}$, Chen $\mathrm{B}$, Wang $\mathrm{H}-\mathrm{Y}$, et al. The efficacy of daily chlorhexidine bathing for preventing healthcare-associated infections in adult intensive care units. Korean J Intern Med 2016;31:1159-70.

$25 \mathrm{Kim}$ HY, Lee WK, Na S, et al. The effects of chlorhexidine gluconate bathing on health care-associated infection in intensive care units: a meta-analysis. J Crit Care 2016;32:126-37.

26 Saint S, Greene MT, Krein SL, et al. A program to prevent catheterassociated urinary tract infection in acute care. N Engl J Med $2016 ; 3374: 2111-9.06 / 02 ; 2020 / 04$

27 Taha H, Raji SJ, Khallaf A, et al. Improving catheter associated urinary tract infection rates in the medical units. BMJ Qual Improv Rep 2017;6:u209593. w7966.

28 IPAC Q. Changing ICU culture to reduce catheter-associated urinary tract infections. Canadian Journal of Infection Control 2018;33:39-43.

29 Reilly L, Sullivan P, Ninni S, et al. Reducing Foley catheter device days in an intensive care unit: using the evidence to change practice. AACN Adv Crit Care 2006;17:272-83.

30 Titsworth WL, Hester J, Correia T, et al. Reduction of catheterassociated urinary tract infections among patients in a neurological intensive care unit: a single institution's success. J Neurosurg 2012;116:911-20.

31 Marra AR, Sampaio Camargo TZ, Gonçalves P, et al. Preventing catheter-associated urinary tract infection in the zero-tolerance era. Am J Infect Control 2011;39:817-22.

32 Blanck AM, Donahue M, Brentlinger L, et al. A quasi-experimental study to test a prevention bundle for catheter-associated urinary tract infections. Journal of Hospital Administration 2014;3:101-8.

33 Davies PE, Daley MJ, Hecht J, et al. Effectiveness of a bundled approach to reduce urinary catheters and infection rates in trauma patients. Am J Infect Control 2018;46:758-63.

34 Clarke K, Tong D, Pan Y, et al. Reduction in catheter-associated urinary tract infections by bundling interventions. Int J Qual Health Care 2013;25:43-9.

35 Sampathkumar P, Barth JW, Johnson M, et al. Mayo clinic reduces catheter-associated urinary tract infections through a bundled 6-C approach. Jt Comm J Qual Patient Saf 2016;42:254-AP4.

36 Reeths A, Merkatoris R. Implementation of external urine collection devices to decrease the standardized utilization ratio of indwelling urinary catheters. Am J Infect Control 2020;48:S33.

37 Rearigh L, Gillett G, Sy A, et al. Effect of an external urinary collection device for women on institutional catheter utilization and catheterassociated urinary tract infections. Infect Control Hosp Epidemiol 2021;42:1-3. 
38 Zavodnick J, Harley C, Zabriskie K, et al. Effect of a female external urinary catheter on incidence of catheter-associated urinary tract 